\title{
PERCUTANEOUS PORTAL VENOGRAPHY
}

\author{
By David Sutton, M.D., M.R.C.P., F.F.R. \\ St. Mary's Hospital, London, W.2
}

Portal venography by percutaneous injection of the spleen was first performed in dogs (Abeatici and Campi, I95I) and the method was later successfully applied in man (Leger, I95 I ; Boulvin et al., 195 I Campi and Abeatici, 1952).

In the last few years the method has been routinely used at a number of centres in the British Isles. Atkinson, Barnett, Sherlock and Steiner (1955) have described about 40 cases investigated at the Hammersmith Hospital. The percutaneous trans-splenic technique is the method most widely used, but some workers prefer portal venography to be performed at laparotomy by injection of a mesenteric vein (Green and du Boulay, 1954). The writer's personal experience is limited to the transsplenic method.

\section{Technique}

We usually perform the investigation under local anaesthesia, the patient being premedicated by a basal narcotic such as omnopon, $\frac{1}{3}$ gr. given one hour before the examination. The patient lies supine and the spleen is punctured in the midaxillary line below the costal margin or between the lower ribs. Large spleens are easy to puncture, but normal-sized or small spleens may prove difficult.

The needles used are of 19 standard wire gauge or of 18 standard wire gauge.

The skin and subcutaneous tissues are infiltrated with local anaesthetic and the splenic puncture is then made through the weal raised in the skin. The needle can often be felt to enter the spleen. If it is correctly sited a backflow of blood is immediately obtained rather like that resulting from a vein puncture. It should be possible to draw back blood easily. Indeed, as most of the cases investigated suffer from portal hypertension, blood will normally drip back through the needle rapidly without any suction being applied to the syringe. In some cases the blood may be at extremely high pressure. The needle is attached to the syringe by a plastic connecting tube and once it is certain that the needle point is correctly sited in the spleen the contrast examination is proceeded with. Atkinson and Sherlock (1954) have, in addition, practised measuring the intrasplenic pressure before proceeding to venography. For the contrast injection 20 to $50 \mathrm{ml}$. of the medium are used and this is injected as rapidly as possible, the procedure being completed within a few seconds. Diodone 50 or 70 per cent. may be used. We have also used the newer tri-iodated organic iodine compounds, such as Diaginol 70 per cent. Rapid serial films are taken, commencing about halfway through the injection of the contrast medium. With a simple cassette tunnel for the hand changing of films, four films can be taken in about six seconds. The needle is kept in the spleen for the minimum period necessary to complete the injection, usually not more than two to three minutes. During this period the patient is warned not to breathe deeply and use only shallow respirations in order to prevent undue movement of the spleen with the needle in situ.

If move elaborate apparatus is available, this, of course, is an advantage. The Schönander biplane apparatus allows films to be taken in two planes and therefore provides even more information, but it should be emphasized that adequate films can be obtained by the simpler method described.

\section{Complications}

Milnes Walker (1954) has reported a case of rupture of the spleen requiring splenectomy three days after a portal venogram. The patient should, therefore, be carefully watched for a few days after the investigation if operation is to be delayed.

The danger of splenic rupture is claimed to be a reason for performing the procedure at laparotomy by Green and du Boulay (1954).

Another risk is that associated with sensitivity to the contrast medium, but this is extremely small, being of the same order as that relating to intravenous pyelograms.

Cases have been recorded where the injection has accidentally been made into the peritoneum, and even into the colon; but these accidents, apart from local pain, have had no serious consequences.

The writer has now injected over 20 cases 
personally. Successful venograms were obtained in all cases and no complications were encountered.

\section{Indications}

The main value of portal venography is in differentiating between intrahepatic and extrahepatic portal obstruction where the diagnosis is clinically difficult. Where this problem arises

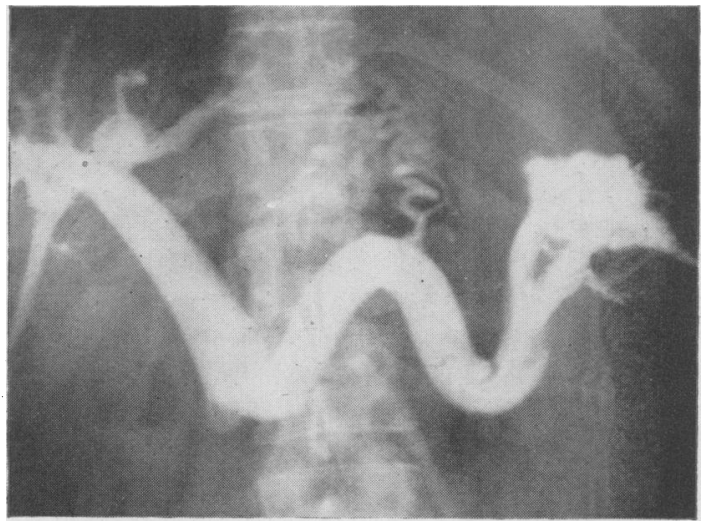

Fig. I.-A case of cirrhosis of the liver. The splenic and portal veins are large in calibre and patent throughout. A porto-caval anastomcsis should be possible. (See Fig. 3). Note the retrograde filling of the inferior mesenteric vein and the gastric varices.

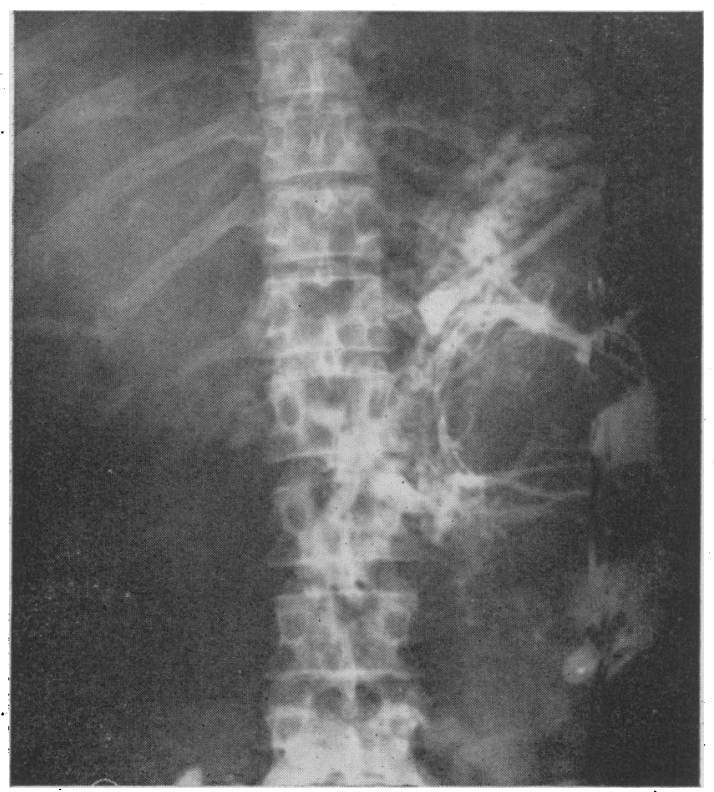

FIG. 2.-The contrast has been successfully injected into the spleen but the splenic vein is thrombosed and the contrast is escaping by means of capsular veins which are forming anastomoses with retro-peritoneal veins and the gastric veins. portal venography will usually provide a clear cự and unequivocal answer.

If surgery is contemplated it will also provide the surgeon with extremely valuable information by demonstrating the size of the portal vein and. whether or not it is possible to anastomose it to the्ह inferior vena cava (Fig. I). It will also show the size and shape of the splenic vein if the alternative operation of a spleno-renal anastomosis is beingconsidered.

Occasionally a case is demonstrated where such? surgical anastomosis is complicated by the presence of splenic vein thrombosis (Fig. 2).

Post-operative splenic venograms are occasion:ally performed to demonstrate the patency of surgical anastomosis (Fig. 3). In one case examined post-operatively by the writer it was though that an anastomosis was functioning well, but the investigation proved conclusively that this was noN the case and that a new portal vein had formed from a dilated periportal vein (Figs. $4^{a}$ and $4 \mathrm{~b}$ ).

\section{Radiological Appearances}

In cases of portal hypertension due to cirrhosis of the liver or to post-hepatic portal obstructiom the splenic and portal veins are usually welf demonstrated, the latter being large in calibre (Fig. I). The collateral circulation is often welf shown also, and where gastric or oesophageado varices are present these may be very well outlined Steiner et al consider that portal venography gifes a better and more accurate demonstration o oesophageal varices than does the simpler examina tion of barium swallow (Fig. 5). The writer hown ever has seen cases with excellent demonstration of oesophageal varices by barium swallow where

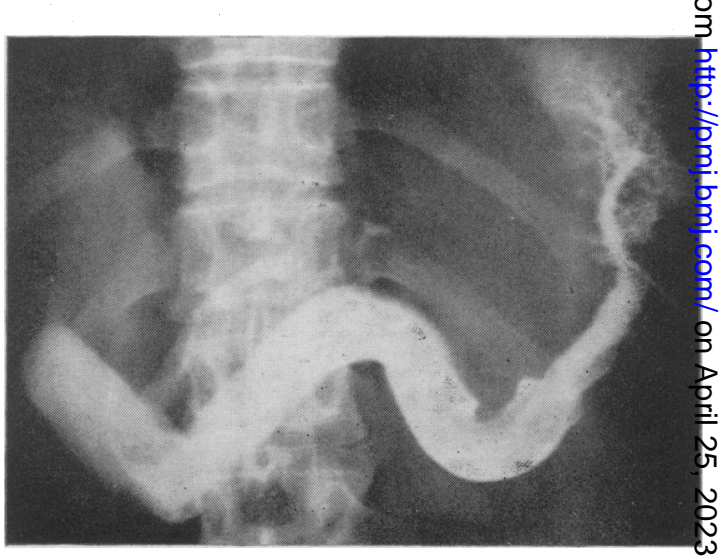

FIG. 3.-A post-operative splenic venogram in the case shown in Fig. 1. The portal vein can now be see emptying into the inferior vena.cava. Note that the needle point actually lies in a main tributary of the splenic vein. The inferior mesenteric vein ando gastric varices shown in Fig. I are no longer seen. 
these have failed to show unequivocally at portal venography. Distortion of the intrahepatic vascular pattern may be seen in cases of cirrhosis, but this may 'be difficult to demonstrate, and is not constantly shown.

The widespread use of percutaneous splenic venography has demonstrated the existence of numerous other collateral channels beside the well recognised ones. Thus capsular veins from the spleen are often shown draining into the retroperitoneal veins to reach the azygos veins (Fig. 2).

Occasionally anastomotic veins are shown in unusual situations and a single huge anastomotic vein may be demonstrated. In such cases opara-

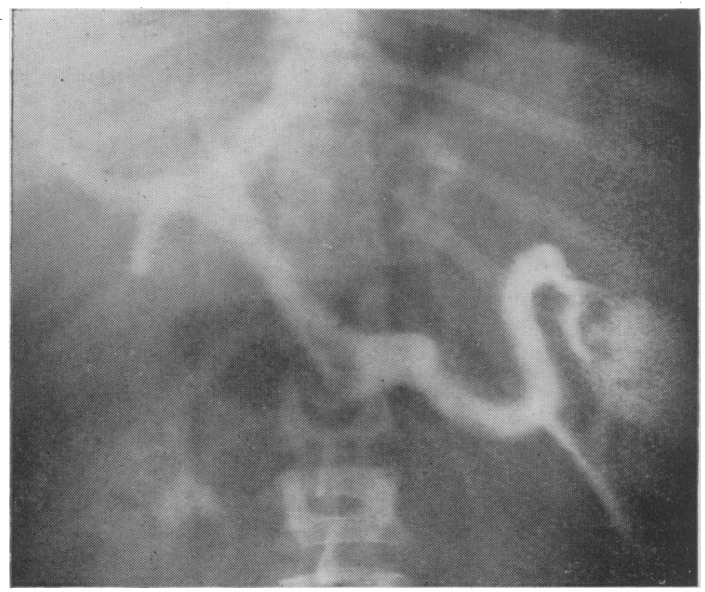

FIG. 4a.-Splenic venogram showing a patent splenic and portal vein in a case of cirrhosis.

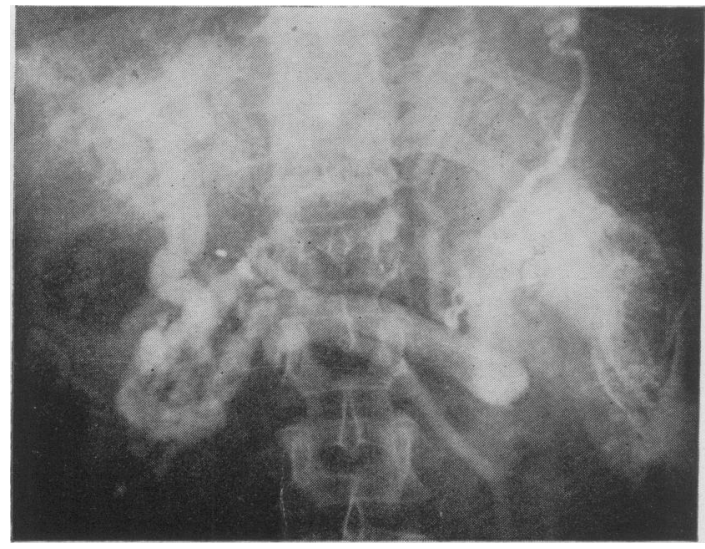

Fic. 4b.-Post-operative venogram following a portocaval anastomosis. The anastomosis has been unsuccessful and the portal tract is filling from enlarged periportal veins. Note the increased number of portal systemic anastomotic veins suggesting increased pressure. tion may be contra-indicated as there already exists a natural 'shunt'.

Doehner et al. (1956) have recently published an excellent survey based largely on post mortem angiographic studies of the collateral circulation in cases of portal obstruction.

In extrahepatic obstruction the portal vein may be seen to be completely thrombosed and the circulation to the liver to be carried by smaller anastomotic veins. The so-called condition of 'cavernoma' of the portal vein, where the latter is replaced by a number of small irregular channels, is probably secondary to thrombosis of the portal vein rather than due to an angiomatous malformation.

In cirrhosis of the liver the position may be complicated by the fact that thrombosis may occur in the portal tract as a secondary complication. Thus even in a known case of cirrhosis it is advisable to have a venogram performed before operation to ensure that the condition is operable. Fig. 2 shows a venogram performed in a woman considered to be suffering from cirrhosis of the liver. In this

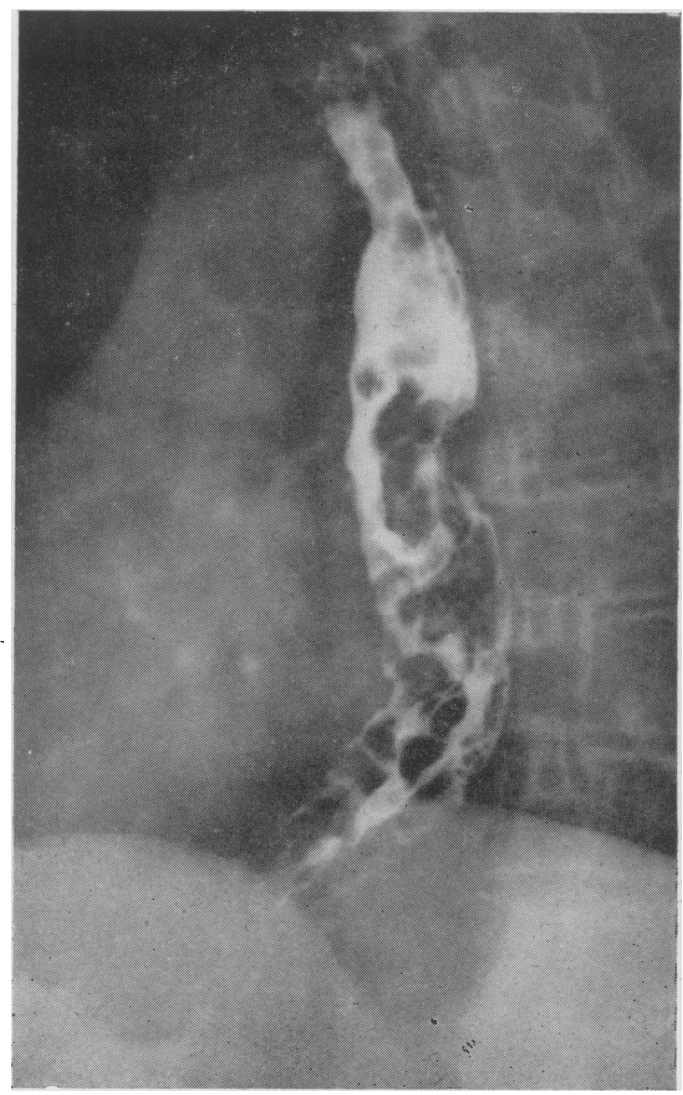

FIG $5 \cdot$-Gastric varices demonstrated by barium swallow. 
case there was no filling of the splenic or portal vein. The splenic vein was thought to be thrombosed its circulation being carried by numerous capsular veins as described above. She was treated surgically by a submucous resection of the varices in the lower oesophagus and by splenectomy. The presence of cirrhosis of the liver was confirmed at laparotomy.

Another similar case has been recently examined and the fact that the portal vein was patent, although the splenic vein was thrombosed, was proved by an operative venogram performed through a mesenteric vein.

\section{Conclusions}

Percutaneous portal venography is now an established method for demonstrating the portal circulation.

The technique is not difficult and the method appears to be safe in competent hands. It provides an excellent method of differentiating betweec intrahepatic and extrahepatic portal obstruction and of demonstrating the site and nature of a extrahepatic block. Prior to surgery it provides the surgeon with useful information as to the operative possibilities. Post-operatively it will demonstrate the patency or inadequacy of a portal shunt. The method therefore is of considerable practical value in the investigation and control of cases of port hypertension.

\section{BIBLIOGRAPHY}

ABEATICI, S., and CAMPI, L. (1951), Acta Radiol., 36, 383. ATKINSON, M., and SHERLOCK, S. (1954), Lancet, i, $1325 . \vec{\circ}$ ATKINSON, M., BARNETT, E., SHERLOCK, S., and STEINER, R. E. (1955), Q. भ. Med., 24, 77.

BOULVIN, R., CHEVALIER, M., GALLUS, P., and NAGEL, 迩 (105 I), Acta chir. Belg., 50, 534.

CAMPI, L., and ABEATIĊI, S. (1952), Radiol. med., 38, I.

DOEHNER, G. A., RUZICKA, F. F., ROUSSELOT, L. M. an HOFFMAN, G. (1956), Radiology, 66, 206.

DU BOULAY, G. H., and GREEN, B. (1954), Brit. F. Radiol., 2 T్ర 423.

LEGER, L. (1951), Mem. Acad. chir., Paris, 77, 7 I 2.

WALKER, R. MILNES. (1954), writing in 'Recent Advances Surgery', ed. by H. C. Edwards, Churchill, London.
The following films have been added to the I.C.I. Film Library :

\section{FILMS ON INDUSTRIAL MEDICINE AND FIRST AID}

\section{Artificial Respiration}

Cat. No. FA. 4. Running time: I I mins. I6mm.

Whatever the cause of asphyxia may be, the condition must be treated by artificial respiration. In this film the Schafer and Holger Nielsen methods are described by the General Chemicals Division medical officer on wall charts, and both methods are then demonstrated by a team of firstaiders on a patient suffering from drowning. The combined method with two operators, and the use of the 'Novox' apparatus is also shown in the film.

\section{Examination of an Unconscious Patient}

Cat. No. FA. 5. Running time: 6 mins. $16 \mathrm{~mm}$.

The object of this film is to teach first-aiders to use a systematic method of examination when they are confronted with an unconscious patient. A first-aider demonstrates this, and the commentary is spoken by a medical officer.

\section{Control of Haemorrhage}

Cat. No. FA 6. Running time: 7 mins. $16 \mathrm{~mm}$.

The theme of this film is that pressure will always control bleeding. The general Chemicals Division medical officer emphasizes this in an introduction to the film, and the various methods of application of pressure are then shown 0 patients by two first-aiders. First, a patient wit arterial bleeding from a wound in the forearm treated, and later, the treatment when a foreige body is present in a wound, the use of ring pads and the important pressure points are demonstra ted.

Removal of Clothing and Treatment of a Fracture Collar-bone

Cat. No. FA. 7. Running time: 6 mins. $16 \mathrm{~mm}^{2}$

A man suffering from a fractured clavicle is: treated by the standard method. Before this can be applied, however, his jacket and waistcoat have to be removed. This film shows in detail the easiest method of removing clothing, followe step by step with the treatment of the fracture.

First Aid for a Patient with a Fractured Spine Cat. No. FA. 8. Running time: 8 mins. I6mm

The victim of an accident involving a fall from height is suspected of having fractured his lumbag spine. A team of first-aiders demonstrates the correct method of handling and treating such af injury, from the initial approach to the eventuas removal of the patient on a stretcher. Th⿱ General Chemicals Division's medical office speaks the commentary and draws attention to the important details of treatment. 